06

\title{
Объемно-температурная зависимость электро- и теплофизических свойств $\alpha$-железа при высоких давлениях и температурах
}

\author{
() А.М. Молодец, А.А. Голышев \\ Институт проблем химической фризики РАН, \\ 142432 Черноголовка, Московская обл., Россия \\ e-mail: molodets@icp.ac.ru
}

Поступило в Редакцию 8 апреля 2021 г.

В окончательной редакции 2 мая 2021 г.

Принято к публикации 3 мая 2021 г.

Представлена модификация полуэмпирического описания объемно-температурной зависимости электропроводности и теплопроводности $\alpha$-железа (железа, имеющего объемно-центрированную кубическую решетку). Рассмотрена интерполяция объемно-температурных зависимостей удельного электросопротивления и коэффициента теплопроводности $\alpha$-железа вдоль высокотемпературных изобар и изотерм высокого давления в диапазоне давлений от -5 до $+5 \mathrm{GPa}$ и температур $400-800 \mathrm{~K}$, соответствующих термодинамическим условиям штатного и аварийного функционирования сплавов железа. Интерполяция электро- и теплофизических свойств $\alpha$-железа выполнена на основе разработанного уравнения состояния $\alpha$-железа при высоких давлениях и температурах. Дана оценка эффекта воздействия сжатия и растяжения на теплопроводность $\alpha$-железа.

Ключевые слова: железо, электропроводность, теплопроводность, уравнения состояния, высокие давления.

DOI: $10.21883 /$ JTF.2021.09.51220.104-21

\section{Введение}

Как известно, железо является основой многочисленных конструкционных сталей. Кроме этого, железо является образцовым веществом в метрологии теплопроводности в диапазоне температур 350-1100 К. Наконец, железо является важным геофизическим металлом, который находится в условиях не только высоких температур, но и высоких (гигапаскальных) давлений. По этим причинам исследованию его теплофизических и термомеханических свойств при высоких давлениях и температурах посвящена обширная научная литература (см. [1-6] и ссылки в них).

Среди теплофизических свойств, прежде всего, представляют интерес теплопроводность и ее зависимость от температуры и давления (или, что то же самое, от объема). Исследования теплопроводности железа, как правило, сопровождаются измерениями его электропроводности. Выяснение закономерностей объемнотемпературной зависимости электропроводности железа, помимо самостоятельного интереса, обусловлено возможностью использованием получаемых данных для расчета электронной составляющей теплопроводности железа на основе закона Видемана-Франца.

Следует отметить, что результаты прецизионных измерений электро- и теплопроводности железа представляются либо в виде табличных массивов данных, либо в виде эмпирических соотношений $[1,2]$ вдоль высокотемпературной атмосферной изобары. Вместе с тем эксплуатация конструкций, а также их функционирование в аварийных ситуациях предполагает возможность расчета теплофизических свойств конструкцион- ных материалов при изменяющихся значениях высоких (до $1000 \mathrm{~K}$ ) температур и высоких (от растягивающих $\sim-5 \mathrm{GPa}$ до сжимающих $\sim+5 \mathrm{GPa}$ ) давлений. Такая задача требует построения физически обоснованных объемно-температурных зависимостей теплопроводности с тем, чтобы можно было рассчитывать значения электро- и теплопроводности железа при целенаправленной вариации давления и температуры. Очевидно, что эта задача тесно соприкасается с определением термомеханических свойств материала таких, как сжимаемость, упругие модули, коэффициент теплового расширения, т.е. с построением адекватного уравнения состояния. Применительно к геофизическим проблемам обычно используются полуэмпирические уравнения состояния полиморфных модификаций железа [3-7].

В настоящей работе с использованием предложенного в [3] модельного соотношения для электропроводности металла и уравнения состояния $\alpha$-железа в форме $[8,9]$ разработаны полуэмпирические соотношения для расчета объемно-температурных зависимостей электро- и теплопроводности $\alpha$-железа в условиях высоких давлений и температур, охватывающих область штатного и аварийного функционирования стальных конструкций.

\section{1. Электрофизические свойства $\alpha$-железа при высоких давлениях и температурах}

Согласно [3], объемно $(V)$-температурную $(T)$ зависимость относительного удельного электросопротивления 
$\eta=\eta(V, T)$ металла при высоких давлениях и температурах можно представить в виде

$$
\eta=\frac{\rho}{\rho_{0}}=\left(\frac{T}{T_{0}}\right)^{\alpha}\left(\frac{V}{V_{0}}\right)^{-n / 3}\left(\frac{\Theta}{\Theta_{0}}\right)^{-n},
$$

где $\rho$ - удельное электросопротивление $\rho=\rho(V, T)$, $\Theta=\Theta(V)$ - зависящая от объема характеристическая температура. В (1) индекс „0“ соответствует априори известным значениям $\rho_{0}, V_{0}, T_{0}$ при начальных условиях (в дальнейшем при атмосферном давлении $P_{0}=1.013 \cdot 10^{-4} \mathrm{GPa}$ и комнатной температуре $\left.T_{0}=300 \mathrm{~K}\right)$. Показатели степеней $\alpha$ и $n$ являются подгоночными параметрами.

Заметим, что соотношение (1) можно преобразовать в явную функцию не только температуры, но и объема, если воспользоваться аналитической функцией для характеристической температуры $\Theta=\Theta(V)$ из [8] (см. также (9) в разд. 3)

$$
\Theta=\Theta_{0}\left(\frac{V_{0}}{V}\right)^{2 / 3}\left(\frac{v_{0}-V}{v_{0}-V_{0}}\right)^{2} .
$$

Действительно, подставляя (2) в (1), получим в отличие от (1) явную функцию не только температуры, но и объема для объемно-температурной зависимости $\eta=\eta(V, T)$ в виде

$$
\eta=\left(\frac{T}{T_{0}}\right)^{\alpha}\left(\frac{V}{V_{0}}\right)^{n / 3}\left(\frac{v_{0}-V}{v_{0}-V_{0}}\right)^{-2 n},
$$

где $\alpha, n-$ те же, что и в (1), параметр $v_{0}$ определяется, согласно [8], через начальный объем $V_{0}$ и начальный коэффициент Грюнайзена $\gamma_{0}=\gamma\left(V_{0}\right)$ как

$$
v_{0}=V_{0}\left(\frac{3 \gamma_{0}+4}{3 \gamma_{0}-2}\right) \text {. }
$$

Таким образом, модификация (3) аналогично (1) содержит два подгоночных параметра, которые определяют объемно-температурную зависимость удельной электропроводности металла, и которые могут быть определены по двум независимым экспериментальным массивам. Так, экспериментальные точки электропроводности вдоль комнатной изотермы высокого давления позволяют найти коэффициент $n$. После этого становится возможным определение коэффициент $\alpha$, если известны экспериментальные значения электропроводности вдоль высокотемпературной изобары. Найдем эти параметры для $\alpha$-железа, опираясь на литературные экспериментальные данные по измерению его электросопротивления.

Заметим предварительно, что, начиная с работ $[10,11]$ по измерению электросопротивления $\alpha$-железа при давлениях до 5-13 GPa, обычно приводятся данные по изменению относительного электросопротивления железных образцов. Для данной работы желательно располагать первичными данными по зависимости от давления удельного электросопротивления. По этой причине ниже отдано предпочтение работе [12], содержащей экспериментальные точки барической зависимости удельного электросопротивления $\alpha$-железа.

Заметим также, что в экспериментах обычно измеряют величину удельного электросопротивления в виде зависимости $\rho=\rho(P, T)$ от давления $P$ или от температуры $T$. Поэтому нахождение значений $n$ и $\alpha$ с использованием модифицированного соотношения (3) предполагает наличие термического уравнения состояния металла $P=P(V, T)$, позволяющее пересчитывать значения $\rho=\rho(P, T)$ в значения $\rho=\rho(V, T)$ вдоль рассматриваемых изотерм $V=V\left(P, T_{\text {const }}\right)$ или вдоль нужных изобар $V=V\left(P_{\text {const }}, T\right)$. К настоящему времени в научной литературе имеются точные полуэмпирические уравнения состояния различных фаз (в том числе и $\alpha$ фазы) железа $[4,5]$. Так, в [5] приведен полный комплект опорных параметров для $\alpha$-фазы железа, который может быть использован для пересчета значений $\rho=\rho(P, T)$ в значения $\rho=\rho(V, T)$. Однако далее воспользуемся термическим уравнением состояния в форме [8], в котором объемная зависимость характеристической температуры в виде (2) является ключевой функцией полуэмпирического описания термодинамики сжатия материала. В контексте настоящей работы этот выбор оправдывается тем, что сочетание (3) с уравнением состояния в форме $[8,9]$ позволяет интерпретировать электрофизические и термодинамические свойства $\alpha$-железа при высоких давлениях и температурах в рамках единого аналитического инструментария. В частности, функция для термического уравнения состояния $P=(V, T)$ в форме [8], представленная формулой (8) (см. разд. 3), позволяет выразить объем в виде $V=V(P, T)$ вдоль комнатной изотермы высокого давления $T=\mathrm{const}=T_{0}$ и преобразовать экспериментальный массив $i$ точек электропроводности [12] с координатами $\rho_{i}=\rho\left(P_{i}, T_{0}\right)$ в точки с координатами $\eta_{i}=\rho_{i}\left(V_{i}, T_{0}\right) \rho_{0}^{-1}$.

Экспериментальные точки [12] в координатах $\eta-V$ показаны точками 1 на рис. 1. Там же представлен график 2 , полученный путем подгонки коэффициента $n$ в (3). Подгонка осуществлялась методом наименьших квадратов. При этом значение $n$ и коэффициент детерминации $R^{2}$ оказались следующими: $n=1.77 \pm 0.02$, $R^{2}=0.992$.

Рассмотрим теперь нахождение второго подгоночного параметра $\alpha$, с привлечением экспериментальных данных по зависимости электросопротивления железа вдоль атмосферной изобары из табл. 4 [1] с величиной $\rho_{0}=\rho_{0 \alpha}=14.4 \mu \Omega \cdot \mathrm{cm}$, которые представлены точками 1 на рис. 2. Предположим, что объемная зависимость относительного удельного электросопротивления для образцов $\alpha$-железа из [12] справедлива и для образцов $\alpha$ железа из [1]. Воспользуемся вновь термическим уравнением состояния и выразим объем $V=V(T, P)$ в виде его зависимости только от температуры $V(T)=V\left(T, P_{0}\right)$ при постоянном атмосферном давлении $P=P_{0}$. Тогда 


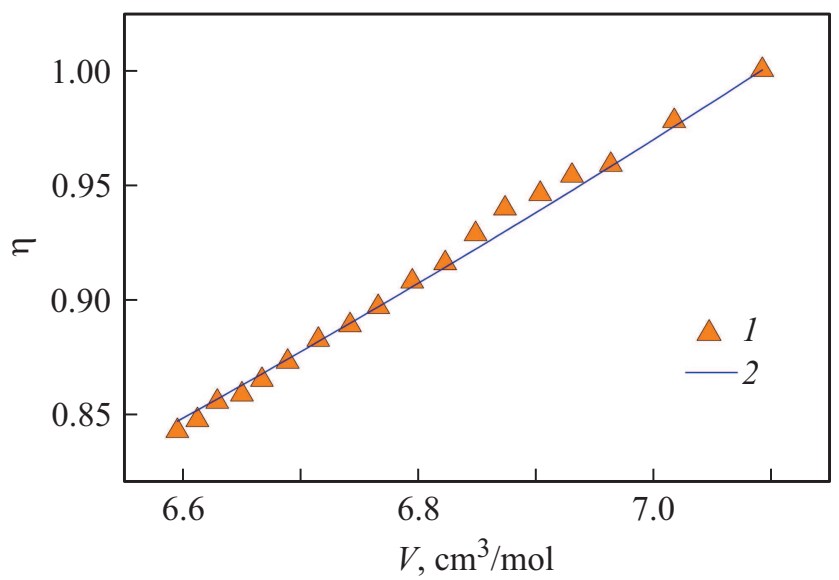

Рис. 1. Зависимость относительного удельного электросопротивления $\alpha$-железа от объема вдоль комнатной изотермы высокого давления: 1 - эксперимент [12] при комнатной температуре $T_{0}, 2$ - подгоночный график (3) при $T=T_{0}$.

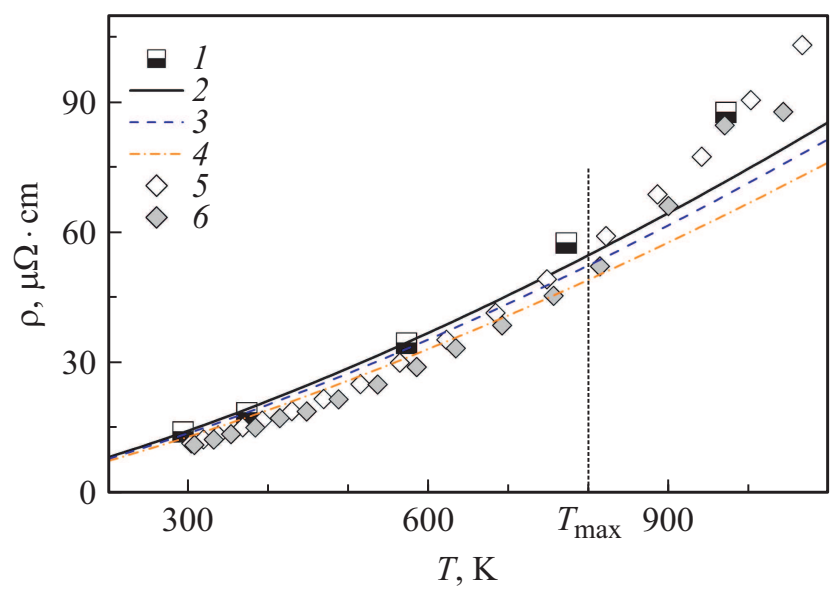

Рис. 2. Зависимость удельного электросопротивления от температуры и давления вдоль изобар $\alpha$-железа: 1 - эксперимент [1] вдоль изобары при атмосферном давлении $P=P_{0}$, 2 - подгоночный график (4) при давлении $P=P_{0}, 3-$ расчетный график (4) вдоль изобары $P=3 \mathrm{GPa}, 4-$ расчетный график (4) вдоль изобары $P=8 \mathrm{GPa}, 5-$ эксперимент [7] вдоль изобары $P=3 \mathrm{GPa}, 6-$ эксперимент [7] вдоль изобары $P=8 \mathrm{GPa}$.

с учетом (3) аппроксимационную функцию температурной зависимости удельного электросопротивления $\alpha$ железа вдоль атмосферной изобары можно записать как

$$
\rho=\rho_{0 \alpha} \eta=\rho_{0 \alpha}\left(\frac{T}{T_{0}}\right)^{\alpha}\left(\frac{V(T)}{V_{0}}\right)^{n / 3}\left(\frac{\nu_{0}-V(T)}{v_{0}-V_{0}}\right)^{-2 n},
$$

где $n=1.77, \alpha-$ искомый подгоночный параметр. График (4) с оптимальным для [1] значением подгоночного параметра $\alpha=1.3$ показан на рис. 2 кривой 2. Как видно, расчетный график 2 согласуется с экспериментом [1] до температуры $T_{\max } \approx 800 \mathrm{~K}$.

Отметим, что комплект величин $\rho_{0 \alpha}, \alpha$ и $n$ вместе с термическим уравнением состояния позволяет
Таблица 1. Параметры соотношений для описания объемнотемпературных зависимостей удельного электросопротивления и теплопроводности $\alpha$-железа

\begin{tabular}{c|c|c|c|c|c|c}
\hline$\rho_{0 \alpha}, \mu \Omega \cdot \mathrm{cm}$ & $\alpha$ & $n$ & $T_{0}, \mathrm{~K}$ & $V_{0}, \mathrm{~cm}^{3} / \mathrm{mol}$ & $\nu_{0}, \mathrm{~cm}^{3} / \mathrm{mol}$ & $k_{0 l}, \mathrm{~W} /(\mathrm{m} \cdot \mathrm{K})$ \\
\hline 14.4 & 1.3 & 1.77 & 298.0 & 7.093 & 21.092 & 21.0
\end{tabular}

провести прогностические расчеты электропроводности железа вдоль изобар высокого давления $V=V\left(P_{\text {const }}, T\right)$ и сравнить их с экспериментом. На рис. 2 представлены такие расчеты для $P_{\text {const }}=3$ и $8 \mathrm{GPa}$ графиками 3 и 4 соответственно. Здесь же приведены экспериментальные данные 5 и 6 из [7] для этих же давлений. Как видно, расчеты 3, 4 и эксперимент 5, 6 практически совпадают друг с другом до температуры $T_{\max } \approx 800 \mathrm{~K}$.

Таким образом, соотношение (4) вместе полуэмпирическим уравнением состояния $\alpha$-железа в форме [8] согласуется с экспериментальными данными по объемнотемпературной зависимости удельного электросопротивления железа в диапазоне температур 300-800 K и давлений 0-8 GРa. Опорные параметры (4) сведены в табл. 1.

Рассмотрим теперь моделирование объемно-температурной зависимости теплопроводности $\alpha$-железа с учетом полученных результатов по электросопротивлению.

\section{2. Теплопроводность $\alpha$-железа при высоких давлениях и температурах}

Привлечем к рассмотрению экспериментальные данные по теплопроводности $\alpha$-железа вдоль атмосферной изобары из [2], мотивируя это тем, что начальное электросопротивление $\rho_{0 \alpha}=14.49-14.68 \mu \Omega \cdot \mathrm{cm}$ исследуемых образцов в [2] практически такое же, как и используемое из [1] в разд. 1. Заметим далее, что вблизи комнатной температуры электронная составляющая теплопроводности образцов железа $k_{e}$, рассчитанная по закону Видемана-Франца $k_{0 e} \approx L T_{0} / \rho_{0 \alpha}$ с использованием теоретического значения числа Лоренца $L=2.45 \mathrm{~W} \cdot \Omega / \mathrm{K}^{2}$, примерно на 30\% меньше экспериментальной. Предполагая, что эта разница целиком обусловлена вкладом решеточной теплопроводности $k_{l}$, запишем выражение для объемно-температурной зависимости теплопроводности железа $k=k(V, T)$ в виде суммы

$$
k=k_{e}+k_{l} \text {. }
$$

В (5) первое слагаемое определяется как $k_{e}=L T / \rho$, где $\rho$ определяется соотношением (4), а второе слагаемое представляет собой модельное выражение объемнотемпературной зависимости решеточной теплопроводности Дугдала-МакДональда $[13] k_{l}=k_{l}(V, T)$

$$
k_{l}=k_{0 l}\left(\frac{T_{0}}{T}\right)\left(\frac{V}{V_{0}}\right)\left(\frac{\Theta}{\Theta_{0}}\right)^{3}\left(\frac{\gamma_{0}}{\gamma}\right)^{2} \text {. }
$$




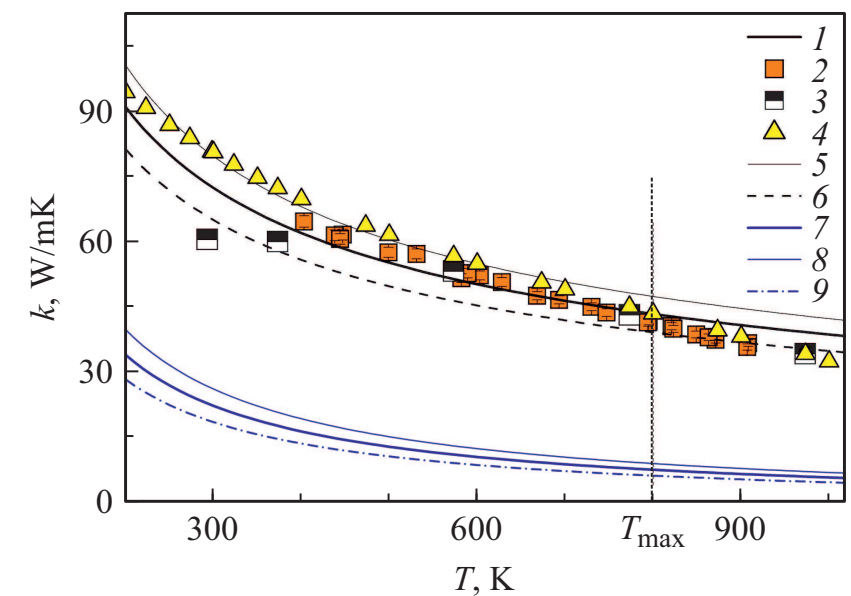

Рис. 3. Зависимость коэффициента теплопроводности от температуры вдоль изобар $\alpha$-железа: 1 - график (5) вдоль изобары при атмосферной давлении, 2, 3, 4 - экспериментальные данные соответственно из $[2,1,14]$ при давлении $P=P_{0}$, 5 - расчетный график (5) вдоль изобары $P=+5 \mathrm{GPa}, 6-$ расчетный график (5) вдоль изобары $P=-5 \mathrm{GPa}, 7-$ расчет решеточной составляющей теплопроводности (6) вдоль атмосферной изобары $P=P_{0}, 8-$ расчет решеточной с оставляющей теплопроводности (6) вдоль изобары $P=+5 \mathrm{GPa}, 9-$ расчет решеточной составляющей теплопроводности (6) вдоль изобары $P=-5 \mathrm{GPa}$.

Функции $\Theta=\Theta(V)$ и $\gamma=\gamma(V)$ в (6) по-прежнему определяются соотношениями (9) и (10) из следующего разд. 3 с описанием используемого термического уравнения состояния $\alpha$-железа. Параметр $k_{0 l}$ является подгоночным параметром, предназначенным для согласования аппроксимационного соотношения (5) с экспериментом.

На рис. 3 графиком 1 представлен расчет $k=k(V, T)$ по (5) вдоль атмосферной изобары с величиной параметра $k_{0 l}=21.0 \mathrm{~W} /(\mathrm{m} \cdot \mathrm{K})$ на фоне литературных данных 2-4 по теплопроводности $\alpha$-железа соответственно из $[2,1,14]$. Как видно, в диапазоне температур 400-800 К модельные расчеты по формуле (5) в комплекте с используемым уравнением состояния совпадают в пределах разброса экспериментальных данных с экспериментом [2 и 1] в пределах 5\%. Модельный график (5) качественно подобен экспериментальной зависимости $k$ из [14], однако количественные различия при температуре $400 \mathrm{~K}$ здесь достигают $13 \%$. Это различие можно соотнести с различием количества примесей в рассматриваемых в [1,2 и 14] образцах.

Очевидно, что соотношение (5) в комплекте с уравнением состояния позволяет рассчитать эффект воздействия сжатия и растяжения на теплопроводность $\alpha$-железа. На рис. 3 представлены расчетные изменения теплопроводности $\alpha$-железа 5 и 6 при сжатии и растяжении соответственно давлениями +5 и $-5 \mathrm{GPa}$. Как видно, эффект воздействия давления на суммарный коэффициент теплопроводности $\alpha$-железа составляет $0.8-1.3 \mathrm{~W} \cdot(\mathrm{m} \cdot \mathrm{K})^{-1}$ на $1 \mathrm{GPa}$. На рис. 3 представ- лены также расчетные изменения коэффициента решеточной теплопроводности $\alpha$-железа при атмосферном давлении 7, при сжатии 8 и растяжении 9. Как видно, эффект изменения решеточной составляющей коэффициента теплопроводности за счет изменения давления в два раза меньше суммарного эффекта и составляет величину $0.3-0.6 \mathrm{~W} \cdot(\mathrm{m} \cdot \mathrm{K})^{-1}$ на $1 \mathrm{GPa}$

Таким образом, модельное представление (5), опирающееся на полуэмпирическое уравнение состояния, согласуется с экспериментальными данными по объемнотемпературной зависимости коэффициента теплопроводности $\alpha$-железа в диапазоне температур $400-800 \mathrm{~K}$ и позволяет оценить изменение коэффициента теплопроводности при воздействии как сжимающих давлений до $+5 \mathrm{GPa}$, так и при воздействии растягивающих давлений до $-5 \mathrm{GPa}$.

\section{3. Термическое уравнение состояния $\alpha$-железа}

Уравнение состояния для $\alpha$-железа строилось на основе полуэмпирического выражения для свободной энергии всесторонне сжатого изотропного твердого тела $F=F(V, T)$, базирующегося на модели эйнштейновских осцилляторов

$$
F=3 R\left[\frac{\Theta}{2}+T \ln \left(1-\exp \left(-\frac{\Theta}{T}\right)\right)\right]+E_{x},
$$

где $R$ - удельная газовая постоянная, $V$ - удельный объем материала, $T$ - его температура, $\Theta-$ объемная зависимость характеристической температуры Эйнштейна, $E_{x}$ - объемная зависимость потенциальной энергии. Функциональный вид обеих функций - упомянутой ранее (см. (2)) $\Theta=\Theta(V)$ и $E_{x}=E_{x}(V)$ - взят из [8].

Термическое уравнение состояния $P=P(V, T)$ (зависимость давления $P$ от объема и температуры $T$ ) определялось как частная производная свободной энергии (7) по объему $P=P(V, T)=-\partial F / \partial V$, которая после дифференцирования приобретает вид

$$
P=3 R \Theta \frac{\gamma}{V}\left(\frac{1}{2}+\frac{1}{\exp (\Theta / T)-1}\right)+P_{x},
$$

где ключевые функции имеют вид явных функций объема:

$$
\begin{gathered}
\Theta=\Theta_{0}\left(\frac{v_{0}-V}{v_{0}-V_{0}}\right)^{2}\left(\frac{V_{0}}{V}\right)^{2 / 3} \\
\gamma=-\frac{d \ln \Theta}{d \ln V}=\frac{2}{3}+\frac{2 V}{v_{0}-V} \\
P_{x}=3 C_{1} x^{1 / 3}\left(-\frac{1}{5} x^{-2}+2 x^{-1}+6-x+\frac{1}{7} x^{2}\right)+C_{2} .
\end{gathered}
$$

В (11) переменная $x=\frac{V}{v_{x}}$ представляет собой удельный объем $V$, нормированный на параметр $v_{x}$, имеющий смысл характеристического объема рассматриваемого 
Таблица 2. Параметры термического уравнения состояния (8) для $\alpha$-железа

\begin{tabular}{c|c|c|c|c|c}
\hline $\begin{array}{c}V_{0}, \\
\mathrm{~cm}^{3} / \mathrm{mol}\end{array}$ & $\begin{array}{c}\Theta_{0}, \\
\mathrm{~K}\end{array}$ & $\begin{array}{c}v_{0}, \\
\mathrm{~cm}^{3} / \mathrm{mol}\end{array}$ & $\begin{array}{c}v_{x}, \\
\mathrm{~cm}^{3} / \mathrm{mol}\end{array}$ & $\begin{array}{c}C_{1}, \\
\mathrm{GPa}\end{array}$ & $\begin{array}{c}C_{2}, \\
\mathrm{GPa}\end{array}$ \\
\hline 7.093 & 279.8 & 21.092 & 21.020 & -144.5188 & 2971.0460
\end{tabular}

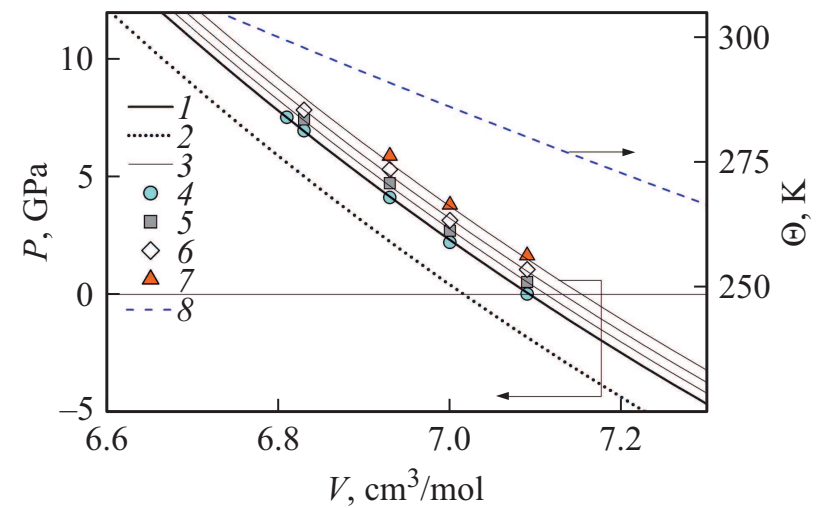

Рис. 4. Объемно-температурные зависимости ключевых функций уравнения (8) для $\alpha$-железа: $1-$ расчетная комнатная изотерма (8), 2 - потенциальное давление (11), тонкие сплошные линии 3 (снизу вверх) - расчетные изотермы соответственно при температурах $300,380,460,550 \mathrm{~K}, 4-7-$ экспериментальные изотермы из [15] при тех же температурах, 8 - объемная зависимость $\Theta=\Theta(V)(9)$.

материала. Используемый комплект определяющих параметров $\alpha$-железа для (8) и их численные значения представлены в табл. 2.

Рис. 4 иллюстрирует степень соответствия термомеханических расчетов и эксперимента при сжатии, а также термомеханическое поведение $\alpha$-железа при растягивающих давлениях до $-5 \mathrm{GPa}$. Здесь 1 - расчетная комнатная изотерма (8), 2 - потенциальное давление (11), тонкие линии 3 снизу вверх - расчетные изотермы соответственно при температурах 300, 380, 460, $550 \mathrm{~K}$. 4-7 - экспериментальные изотермы из [15] при этих же температурах 300, 380, 460, 550 К. Как видно, расчетные высокотемпературные изотермы $\alpha$-железа совпадают с высокотемпературными экспериментальными изотермами [15] в пределах разброса экспериментальных данных. На рис. 4 для справки показан также график 8 объемной зависимости $\Theta=\Theta(V)$, посчитанной по формуле (9) в области сжимающих и растягивающих давлений.

Таким образом, расчеты термодинамического состояния $\alpha$-железа с использованием термического уравнения состояния в форме (8) согласуется с экспериментом в диапазоне давлений 0-8 GPa и температур 300-540 K, что оправдывает использование этого уравнения состояния для описания экспериментальных электро- и теплофизических данных, предпринятое в предыдущих разделах настоящей работы.

\section{Заключение}

Подытоживая разработанное полуэмпирическое описание экспериментальных данных по электро- и теплофизическим свойствам $\alpha$-железа, можно сказать, что модифицированное соотношение для объемнотемпературной зависимости удельного электросопротивления металлов вместе с авторским термическим уравнением состояния представляет собой единообразное и адекватное описание экспериментальных данных по объемно-температурной зависимости удельного электросопротивления и коэффициента теплопроводности $\alpha$ железа вдоль атмосферной изобары в диапазоне температур $300-800 \mathrm{~K}$.

Аналитический инструментарий представленного полуэмпирического описания позволяет рассчитывать температурную зависимость электросопротивления $\alpha$ железа при сжатии давлениями до $8 \mathrm{GPa}$, а также оценивать изменение коэффициента теплопроводности $\alpha$-железа при воздействии давлений от сжимающих $+5 \mathrm{GPa}$ до растягивающих $-5 \mathrm{GPa}$. Эффект воздействия давления на суммарный коэффициент теплопроводности $\alpha$-железа составляет $0.8-1.3 \mathrm{~W} \cdot(\mathrm{m} \cdot \mathrm{K})^{-1}$ на $1 \mathrm{GPa}$. Эффект изменения решеточной составляющей коэффициента теплопроводности за счет изменения давления составляет величину $0.3-0.6 \mathrm{~W} \cdot(\mathrm{m} \cdot \mathrm{K})^{-1}$ на $1 \mathrm{GPa}$.

\section{Финансирование работы}

Работа выполнена по теме Государственного задания № AAAA-A19-119071190040-5.

\section{Конфликт интересов}

Авторы заявляют, что у них нет конфликта интересов.

\section{Список литературы}

[1] П.П. Олейников. ТВТ, 19 (3), 533 (1981).

[2] Е.С. Амасович, В.Э. Пелецкий. ТВТ, 20 (5), 891 (1982).

[3] C.T. Seagle, E. Cottrell, Y. Fei, D.R. Hummer, V.B. Prakapenka. Geophys. Res. Lett., 40, 5377 (2013).

[4] A. Dewaele, P. Loubeyre, F. Occelli, M. Mezouar, P.I. Dorogokupets, M. Torrent. Phys. Rev. Lett., 97, 215504 (2006).

[5] P.I. Dorogokupets, A.M. Dymshits, K.D. Litasov, T.S. Sokolova. Scientific Reports, 7, 41863 (2017). DOI: $10.1038 /$ srep41863

[6] C. Zhang, J.-F. Lin, Y. Liu, S. Feng, C. Jin, M. Hou, T. Yoshino. J. Geophys. Research: Solid Earth, 123, 3564 (2018).

[7] R.E. Silber, R.A.Secco, W. Yong, J.A.H. Littleton. J. Geophys. Research: Solid Earth, 124, 5521 (2019).

[8] А.М. Молодец, Д.В. Шахрай, А.А. Голышев. ТВТ, 55 (4), 523 (2017).

[9] А.М. Молодец. ФТТ, 55 (11), 2090 (2013).

[10] P.W. Bridgman. Proceed. American Academy of Arts and Sciences, 52 (9), 573 (1917).

[11] A.S. Balchan, H.G. Drickamer. Rev. Sci. Instrum., 32 (3), 308 (1961). 
[12] D. Jaccard, A.T. Holmes, G. Behr, Y. Inada, Y. Onuki. Phys. Lett. A, 299 (2-3), 282 (2002).

[13] J.S. Dugdale, D.K.C. MacDonald. Phys. Rev., 98 (6), 1751 (1955).

[14] C.Y. Ho, R.W. Powell, P.E. Liley. J. Phys. Chem. Ref. Data, 1 (2), 279 (1972).

[15] S. Klotz, Th. Strässle, A.L. Cornelius, J. Philippe, V. Pomjakushin. J. Phys. D: Appl. Phys., 44, 055406 (2011). 